\title{
Preface to the special issue on earthquake geodesy*
}

\author{
Sidao $\mathrm{Ni}^{*}$ \\ CAS Key Laboratory of Dynamical Geodesy, Institute of Geodesy and \\ Geophysics, Wuhan 430077, China
}

Geodesy is among the oldest branches of Earth science with historical record of geodesy study by Thales of Miletus more than 2000 years. Originally geodesy was defined as the science of measuring and portraying of the Earth's surface, i.e., assuming that the Earth is not changing with fixed shape. But in the past decades geodesy has evolved into a branch of Earth science that focuses on studies of a dynamic Earth. By monitoring and modeling the changes of the Earth's shape (geokinematic), gravity field and rotation (i.e., the three pillars of geodesy, IAG-GGOS documents), geodesy has been the most broadly applied area of Earth science.

In contrast, seismology, originally defined as the study of Earth's vibration, has always been dealing with a dynamic Earth with earthquakes as the most impressive dynamic feature. Seismology is also an old branch of earthquake science, for example, about 2000 years ago, Heng Zhang of Han Dynasty in China invented the first instrument responding to an earthquake. But only after the great Lisbon earthquake in 1755, seismology began to evolve into a modern science. Though both geodesy and seismology study a dynamic Earth, they used to cover different aspects of Earth's motion. Geodesy is more involved with slow motions (months, years and longer) with large displacements (millimeters and above), while seismology is more concerned with rapid motion (tens of seconds to tens of hertz) with small displacements (millimeters and less, for broadband seismology).

However, with advent of precise spatial geodesy such as GPS and InSAR as well as accurate ground geodetic instrumentations such as VLBI and superconducting gravimeters, geodesy is able to observe Earth motions in the seismic band. For example, high rate GPS can be effectively used as precise instrument recording dynamic displacement due to earthquakes with frequency content up to a few $\mathrm{Hz}$ (Avallone, 2011). Moreover, GPS and gravimeters provide accurate mea- surement of static co-seismic displacement which is not achievable with seismometers. More impressively, InSAR provides information about the displacements of an earthquake for a large area, making it possible to resolve ground truth location and detailed fault geometries of earthquakes (Massonnett et al., 1993). Due to the extended capability of observing earthquakes with modern geodesy, a new branch of science of interdisciplinary study between geodesy and seismology has come into being, i.e., earthquake geodesy.

Earthquake geodesy is essentially the science of observing and modeling of earthquake processes (interseismic, co-seismic and post-seismic) as well as resolving internal structure of the Earth with geodetic approaches. This special issue covers recent advances in studies of earthquake geodesy, including both studies of earthquake processes and structure of the Earth. Wang et al. tackled the difficult problem of resolving crustal density structure by joint inversion of gravity and seismic travel time data. Though body waves and surface waves provide high definition of velocity structure of the Earth, seismology is particularly insensitive to density structure of the earthquake. By joint inversion of gravity and seismic data, Wang et al. were are able to get a better image of the crustal density structure, thus leading to better understanding of dynamic process in North China.

High rate sampling gravity data can also be used to study velocity structure of the Earth. Zeng et al. demonstrated that Green's function can be retrieved from correlation between seismic data and gravity data, which is usually achieved from correlation between seismic data. As gravity data features lower noise in long period (period of hundreds of seconds to dozens of seconds) as compared to seismic records, it can be expected that Green's functions from gravity records can provide valuable constraints on deeper structure of the Earth. As the most accurate gravity instrument, super- 
conducting gravimeter provides high quality records for studying free oscillation of the Earthquake (with period of hundreds of seconds, up to thousand seconds), and Lei et al. analyzed such dataset to provide constraint on very deep structures such as the lower mantle and the core.

Geodetic observation provides key constraints on studies of whole earthquake cycles, including interseismic, co-seismic and post-seismic processes. For example, by modeling the GPS-observed motion o f tectonic units in Tibet, Zheng et al. found that faults play important role in tectonic evolution of Tibet. Other geodetic techniques such as GPS and InSAR are crucial for understanding post-seismic processes. Shao et al. studied the rapid post-seismic deformations from the 2008 great Wenchuan earthquakes, and found that both afterslip and viscoelastic relaxation are needed to explain the observed spatial distribution of post-seismic deformation. Assuming a viscoelastic crustal model, Li$\mathrm{u}$ et al. predicted co-seismic and post-seismic deformation and gravity change associated with the 2010 Yushu earthquake in western China. Geodetic methods are also valuable for studying earthquake rupture processes. With high quality InSAR observation of deformation field, Li et al. obtained high resolution model of the 2009 Fiordland earnthquake in New Zealand. And Wei et al. performed joint inversion of InSAR and teleseismic body waves to resolve rupture processes of the 2004 Zhongba earthquake in Tibet.

While geodesy contributes substantially to seismology, seismological studies also help geodesy. Chen et al. showed that shallow shear velocity structure in sedimentary basin brings bias in GPS or InSAR observation of ground displacements by a factor up to two. Bao et al. proved that precise location of aftershocks combined with InSAR inversion is crucial to studies of whether rupture area of two main shocks overlap. Similarly, Xie et al. demonstrated that combination of InSAR and seismic location with ambient noise is necessary for nailing down the ground truth location for strong earthquakes (InSAR is applicable because of large deformation involved) and small earthquakes where seismological methods are more applicable.

In Summary, as the interdisciplinary study between seismology and geodesy, earthquake geodesy is a new and promising field of research. It can be expected that most major earthquakes in the future will be studied in this interdisciplinary approach.

Last but not least, I want to express gratefulness to all the authors for their interesting work and the reviewers for their kind help. Also special thanks to Ms. Tian and Ms. Liu for their patience and professionalism as editors.

\section{References}

IAG-GGOS, http://www.iag-ggos.org/about_geodesy/the_ three_pillars.php, IUGG meeting in Sapporo.

Avallone A, Marzario M, Cirella A, Piatanesi A, Rovelli A, Di Alessandro C, D'Anastasio E, D'Agostino N, Giuliani $\mathrm{R}$ and Mattone $\mathrm{M}$ (2011). Very high rate $(10 \mathrm{~Hz}) \mathrm{GP}-$ $\mathrm{S}$ seismology for moderate-magnitude earthquakes: The case of the $M_{\mathrm{W}} 6.3$ L'Aquila (central Italy) event. $J$ Geophys Res 116: B02305, doi:10.1029/2010JB007834.

Massonnet D, Rossi M, Carmona C, Adragna F, Peltzer G, Feigl K and Rabaute T (1993). The displacement field of the Landers Earthquake mapped by Radar Interferometry. Nature 364(6 433): 138-142. 